\title{
Potential and limits of InSAR data for building reconstruction in built-up areas
}

\author{
U. Stilla*, U. Soergel, U. Thoennessen \\ FGAN-FOM Research Institute for Optronics and Pattern Recognition, \\ D-76275 Ettlingen, Germany
}

\begin{abstract}
The automatic reconstruction of buildings for the generation of city models is of great interest for different tasks. 3D information can be directly obtained from both, laser (LIDAR) and radar (InSAR) measurements. The features of both sensors are compared. The data acquisition by SAR is described, with emphasis on the special properties of the interferometric SAR principle. A segmentation approach for building reconstruction is proposed. The results show that building reconstruction is possible from InSAR, but the achievable level of detail cannot compete with LIDAR. The main source of limitation is the inherent side-looking scene illumination of SAR, giving rise to disturbing phenomena interfering with often large parts of the scene. Geometric constraints for the location and size of such problem areas are derived. In order to identify areas of unreliable data in SAR images of a built-up area corresponding elevation data are analysed. The impact of the phenomena layover, shadow and dominant scattering at building locations is considered. For this task a hybrid elevation reference is required. The buildings and the surrounding ground are represented as CAD planes. Natural objects like trees and bushes remain in the raster representation.
\end{abstract}

Keywords: interferometric SAR; LIDAR; built-up areas; building reconstruction

\footnotetext{
* Corresponding author: Tel.: +49 7243 992-328; Fax: +49 7243 992-299.

E-mail address: stilla@fom.fgan.de (U. Stilla).
} 


\section{Introduction}

Three-dimensional city models are of great interest for visualisation, simulation and monitoring purposes in different fields. A typical application is the visualisation of the influence of a planned building to the surrounding townscape for city and regional planning. Additionally, there is a growing demand for such models in civil and military mission planning. City models are used as basis for simulation e.g. in the fields of environmental engineering for microclimate investigations or telecommunications for transmitter placement. Furthermore, 3D information can be used for monitoring, e.g. damage assessment after an earthquake.

All the mentioned tasks require knowledge about the 3D structure of buildings. Besides the classical photogrammetric approaches to determine object heights indirectly, range sensors like LIDAR and interferometric SAR (InSAR) have played an important role since recent years. Table 1 gives an overview of some features of both systems.

(Table 1)

For topographic mapping data acquisition in nadir view is advantageous, especially in dense urban scenes with elevated objects. The LIDAR principle allows airborne applications in oblique (e.g. obstacle warning systems) and in nadir view as well. In contrast to LIDAR the SAR principle requires a side-looking illumination. LIDAR is based on the time-of-flight measurement of reflected infrared laser pulses, while InSAR exploits the phase difference of two measurements of the radar cross-section. Current laser systems achieve higher elevation 
accuracy compared to radar systems which are more sensitive to noise, because the measurement is based on a phase difference of two signals. Typically, the obtained accuracy is in the order of dm for LIDAR and a few meters for InSAR. LIDAR and InSAR are both active systems, which illuminate the scene with electromagnetic waves and measure the backscattered signal component. Due to the different wavelengths (e.g. LIDAR: $1.5 \mu \mathrm{m}$, SAR: $3 \mathrm{~cm}$ ) special surface properties are sensed, because the reflectance depends mainly on the surface roughness compared to the wavelength. Furthermore, different behaviour concerning atmospheric attenuation and weather conditions are observed. While radar shows almost no sensitivity to weather influence, laser is attenuated from rain or fog and the signal might be reflected away from the sensor if the surface is covered with frost or ice. Another advantage of SAR is the opportunity of recording large areas in a short time and from a large distance. Fig. 1 shows a SAR image from a section of the test area Karlsruhe $(1$ pixel $=1 \mathrm{~m} x$ $1 \mathrm{~m})$.

(Figure 1)

Due to the mentioned features, both sensors are of interest for the generation of 3D descriptions (Gamba and Houshmand, 2000). For some applications, e.g. disaster management LIDAR data (Kakumoto et al., 1997) as well as InSAR data (Takeuchi et al., 2000) were evaluated. A fusion of LIDAR and InSAR data of vegetated areas was proposed by Slatton et al. (2000).

This paper is organized as follows. First the SAR and InSAR principles are recapitulated with emphasis on the illumination phenomena. Then an approach for a building segmentation in InSAR data is proposed. A building reconstruction in dense built-up areas is often hindered 
by mutual interference of the signal from the buildings by layover, shadow and strong scattering effects. Geometric constraints for the location and size of such problem areas are derived for a given sensor altitude and viewing angle. If reference elevation data is available it is useful to study the influence of e.g. different aspect angles of the SAR acquisition on the location and size of such areas. With respect to the building reconstruction task it is important to determine the portion of undisturbed measurable building roof areas. In order to consider the influence of man-made objects as well as natural objects a hybrid ground truth (raster and vector data) is chosen.

\section{Elevation data from radar}

\subsection{Acquisition of SAR data}

The moving sensor illuminates the scene with microwaves in a side-looking manner. The signal is partly reflected away from the sensor, scattered towards the sensor, or absorbed, depending on properties of the soil like the roughness compared to the signal wavelength and the dielectric constant $\varepsilon$. The sensor wavelength (e.g. X-band: 3cm, P-band: $64 \mathrm{~cm}$ ) should be chosen with respect to the given task. The test data were acquired with the airborne AER-II sensor of FGAN (Ender, 1998). AER-II is equipped with an active, fully polarimetric phased array antenna operating at a center frequency of $10 \mathrm{GHz}$ (X-band). The signal bandwidth is $160 \mathrm{MHz}$. Four channels are available to record simultaneously either the polarimetric combinations (HH, HV, VV, VH) or two interferograms (e.g. HH and HV).

\subsection{Interferometric SAR principle}


SAR interferometry takes benefit from the coherent SAR measurement principle. Fig. 2 illustrates the principle of airborne single-pass across-track interferometry measurements. Two antennas are mounted above each other on the carrier with geometric displacement B. One of the antennas illuminates the scene and both antennas receive the backscattered complex signals (single baseline mode).

(Figure 2)

The interferogram $S$ is calculated by a pixel by pixel complex multiplication of the master signal $s_{1}$ with the complex conjugated slave signal $s_{2}$. These signals can be expressed in polar coordinate notation $s=a \cdot e^{j \varphi}$ with amplitude a and phase $\varphi$. Due to the baseline $\mathrm{B}$, the distances from the antennas to the scene differ by $\Delta \mathrm{r}$, resulting in the phase difference $\Delta \varphi$ of the interferogram:

$S=s_{1} \cdot s_{2}^{*}=a_{1} \cdot e^{j \varphi_{1}} \cdot a_{2} \cdot e^{-j \varphi_{2}}=a_{1} \cdot a_{2} \cdot e^{j \Delta \varphi}$

with $\Delta \varphi=-\frac{2 \pi}{\lambda} \Delta r$ and $\lambda$ wavelength.

The phase difference $\Delta \varphi$ is unambiguous in the range ]- $\pi, \pi]$ only. Thus, a phase-unwrapping step is often required before further process. Furthermore, the range dependency of $\Delta \varphi$ has to be removed (flat earth correction). Afterwards, the elevation differences $\Delta \mathrm{h}$ in the scene can be derived from $\Delta \varphi$ :

$$
\Delta h \approx \frac{\lambda}{2 \pi} \cdot \frac{r \cdot \sin (\theta)}{B \cdot \cos (\xi-\theta)} \cdot \Delta \varphi,
$$


with parameters distance $r$, antenna geometry angle $\xi$ and viewing angle $\theta$ (off nadir). The coherence $\gamma$ is a function of the noise impact of the interferogram. It is usually locally estimated from the data by the magnitude of the complex cross-correlation coefficient of the SAR images. For each pixel of the interferogram the coherence is calculated using a window with $\mathrm{N}$ pixels:

$$
\hat{\gamma}=\frac{\left|\sum_{n=1}^{N} s_{1}^{(n)} \cdot s_{2}^{(n)^{*}}\right|}{\sqrt{\sum_{n=1}^{N}\left|s_{1}^{(n)}\right|^{2} \cdot \sum_{n=1}^{N}\left|s_{2}^{(n)}\right|^{2}}} \approx \frac{1}{1+\frac{1}{S N R}}
$$

Hence, the local quality of an InSAR DEM can be directly assessed from the data by the related coherence value. Furthermore, the coherence is exploited for change detection tasks: changes in the period between repeated SAR measurements lead to decorrelation of the signals.

\subsection{Geometric interpretation}

Particularly in urban areas, phenomena like layover, shadow, multi-path signals and speckle (Schreier, 1993) have to be considered. Some of the phenomena are illustrated in Fig. 3.

(Figure 3)

Let us assume a building is sensed in a side looking manner from point $\mathrm{F}$. In the slant range image IS the points A, B, C appear according to their distance to the sensor (Fig. 3a). Hence, 
the point $\mathrm{A}$ on the corner line of the building appears as $\mathrm{A}^{\prime}$ behind point $\mathrm{B}^{\prime}$ and in-between $\mathrm{B}^{\prime}$ and C'. The area B'A' is called layover area. Layover occurs always at vertical building walls facing towards the sensor. It leads to a mixture of signal contributions from the building and the ground in the SAR image, because the elevated objects are closer to the sensor than the ground. The slant image shows a geometric distortion in range direction which makes object recognition and interpretation more difficult. For image interpretation the data are usually sampled to a rectangular grid on the ground (Fig. 3b). However, the order of the points B", A", C" in the ground image is still the same as in the slant image. On the other side the building casts a shadow which occludes smaller objects behind and appears dark in the image. However, the height of a detached building can be derived from the shadow length and the viewing angle.

\section{Segmentation of InSAR data}

In our approach the entire set of complex InSAR data (phase, intensity, and coherence) is analysed for the segmentation of extended buildings. An overview of the segmentation process is given in Fig. 4. In a pre-processing step, the intensity data is despeckled and the elevation information is smoothed by median filtering. Fig. 4 (left, top) shows the intensity image (ground range) of a scene section of Frankfurt airport. The range direction is from top to bottom and the ground resolution is approximately $1 \mathrm{~m}$. Corresponding to this image the elevation and coherence is shown. Several extended buildings of the airport cargo centre are present in the scene. The rooftops are generally flat with small elevated superstructures, mostly required for illumination and air-conditioning inside the building. 
(Figure 4)

\subsection{Generation of a depth map considering the SNR}

The initial segmentation is carried out by a combined region growing in the intensity and the elevation data (Soergel et al., 2000). The threshold of region growing is set to a small value in order to detect as many object boundaries as possible. As a consequence, over-segmentation occurs, which is corrected in a subsequent post-processing step. In case of flat roof structures and a constant SNR in the scene the average elevation of the roof is the maximum likelihood estimate (MLE) of the building height. But the SNR varies in the scene even inside the roof boundaries. In order to consider this variation the elevation samples are weighted with the coherence for the averaging step. This results in a preliminary depth map of prismatic objects. Segments with low average intensity and coherence are regarded as unreliable. These segments are assumed to coincide with shadow areas or roads and are considered later to check the consistency of the results.

\subsection{Detection of buildings}

Elevated segments which match a building model according to size and shape are considered as building candidates. Shadow cast from a rectangular flat building part leads to either long or L-shaped segments, depending on the aspect. Their width is a function of viewing angle and object height. Hence, for each building candidate an expectation area for a shadow stripe is predictable. Unfortunately, shadow cannot always be distinguished from objects which appear similar in the data, like roads. Therefore, as a minimum requirement, an area of the set of unreliable segments is expected to be found at the predicted shadow location. If so, the 
candidate segment is labeled to be a building. In case shadow does not interfere with roads, a more subtle analysis is carried out. Shadow stripes are extracted in the intensity data with a simple structural image analysis algorithm.

\subsection{Post-processing}

Shadow areas are used to overcome under-segmentation. Segments containing a possible shadow area are further investigated (Soergel et al., 2000). The under-segmentation is corrected in two different ways. If the histogram of the original height values shows a bimodal curve, the segment is split in two closed segments, if possible. In a second approach a region-growing step in the median filtered heights is carried out. In contrast to the initial segmentation, the border towards the shadow region is used as seed (Hoepfner, 1999) and the threshold is smaller. Over-segmentation is corrected by merging adjacent segments with similar heights. After post-processing the depth map is recalculated.

\section{Limitations of building reconstruction from SAR and InSAR}

It was shown that in InSAR data a segmentation of building structures is possible. The InSAR test dataset Frankfurt contained large and detached buildings with flat roofs. Extended building parts with different height could be distinguished by the segmentation approach. Due to the noisy nature of the InSAR elevation data, the achieved level of detail was not comparable with results from LIDAR (e.g. Stilla and Jurkiewicz, 1999). 
The smoothed InSAR elevation data can be used for change detection purposes, at least for extended buildings. But the results indicate that the approach is limited to coarse scene descriptions only. For image based detail analysis, like roof reconstruction, the data is still too inaccurate. If a further improvement of the accuracy is achievable more sophisticated object models become appropriate in the future. The segmentation results might be used to correct the geometric displacement of the buildings. Furthermore, they could be incorporated in a refined phase unwrapping step in which shadow and layover areas are masked.

The reconstruction of man-made objects in dense urban areas from SAR imagery is limited by inherent geometric constraints. The mentioned effects of layover and shadow (explained in Fig. 3) are visible in Fig. 5.

(Figure 5)

A part of the scene covered by the SAR image of Fig. 1 contains a high building (see Fig. 5a). The corresponding section of the SAR image was rotated by $90^{\circ}$ and is displayed in Fig. $5 \mathrm{~b}$ (illuminated from right to left). For comparison, a nadir view of the scene section is shown by an aerial image in Fig. 5c and the building layer of a digital vector map is overlaid (Fig. 5d). Due to the elevation, the building appears shifted towards the sensor, covering partly a smaller building. Additionally, a building is partly occluded from the shadow cast by the high building.

As shown in Fig. 2b, layover (Area R1) leads to a signal mixture from ground and building (wall and roof), in contrast to area R2, which origins from the roof only. The width (A"C") of 
area R2 depends on angle $\theta$ and the building geometry $\mathrm{w}$ and $\mathrm{h}$. R2 disappears, if $\mathrm{h}=\mathrm{w} \tan \theta$ when the point $C$ falls together with point $A$ on the ground (Fig. 6a).

(Figure 6)

That means, if $\mathrm{h}=\mathrm{w} \tan \theta$, a proper roof reconstruction is not possible anymore. As a consequence, for building reconstruction a large viewing angle $\theta$ seems to be advantageous. However, a large $\theta$ leads to extended shadow regions behind buildings, which might occlude a lower building or street of width $s=h \tan \theta$ (Fig. 6b). Furthermore, the area of unreliable image data should be extended from layover $l$ caused by buildings located at the other side of the street, as depicted in Fig. 6c.

At building walls, multi-path propagation of signals occurs, which leads to a wrong range. Double-bounce propagation at the extended dihedral corner reflector between the ground and the building wall is mapped to the location at the building footprint (see Fig. 3). This leads to a line of bright intensity in azimuth direction at the edge of the building wall and ground.

Occlusion mainly from trees limits the presence of double-bounce scattering to a few locations only in Fig 5. There is such an event between the high building and the flat roof of the smaller building in front. Hence, the line of bright scattering appears shifted towards the sensor and not at the footprint of the high building, which would be the location in case of double-bounce between wall and ground. 
Additionally, roofs with perpendicular orientation towards the sensor cause total reflections and strong signal responses (Fig. 6d). Corners and sloped rooftops may lead to dominant scattering due to multi-bounce effects, respectively perpendicular orientation towards the sensor, depending on the aspect (e.g. at the circle around the castle in Fig. 1).

\section{Detection of SAR phenomena incorporating elevation data}

\subsection{Detection of shadow and layover}

Because of the phenomena mentioned above the radar illumination aspect is important. Hence, the data acquisition of certain areas of the urban scene may be optimised by a simulation of SAR phenomena. A suitable basis for such a simulation (Soergel et al., 2002a) is a 3D city model, which can be obtained from LIDAR (Fig. 7a).

(Figure 7)

For the detection of layover and shadow areas an approach proposed for satellite SAR data and DTM data (Meier et al., 1993) was adopted to high-resolution LIDAR DEM. The idea is to determine the part of the scene which can be seen reliably from a given sensor position. The analysis is restricted to the geometric alignment of the scene towards the sensor, neglecting the influence of the antenna side-lobes and the aspect variation over the synthetic aperture. The elevation grid is sampled incoherently in range direction. For each bin the distance and viewing angle towards the sensor are calculated, which are analysed to detect shadow and layover areas. 
The use of a coarse DTM restricts the approach to the macroscale level (terrain slope), which is often sufficient for satellite data. In order to analyse high-resolution SAR data of urban scenes, the mesoscale level (e.g. buildings) has to be considered as well. For the study of shadowing and layover at building locations elevation information can be derived e.g. from CAD building descriptions (Bolter and Leberl, 2000).

Even in dense urban areas, trees may cover large portions of the terrain. Neglecting the influence of natural objects might result in too optimistic estimations of the visibility of manmade objects. In this paper, LIDAR data was chosen as ground truth elevation information to take trees into account. First, pulse data was used because the SAR image was acquired in the $\mathrm{X}$-band (HH polarization, 3 looks). The ground resolution is approximately $1 \mathrm{~m}$, off nadir angle was about 55 degree and sensor altitude over ground $3 \mathrm{~km}$. Range direction is from north to south (Fig. 1).

The calculated shadow areas are shown in Fig. 7b in black and layover areas are shown in Fig. 7c in white. A combined visualisation of both effects is illustrated in Fig 7d. Layover is depicted in white, shadow in black, and areas with a mixture of layover and shadow appear dark grey. The region of the scene which is expected to lead to undisturbed signal is represented in bright grey. In Fig. 7e, the corresponding section of the SAR image which was acquired from the simulated position is depicted for comparison.

The results of the shadow/layover detection are given in Table 2. According to the viewing angle one might expect a larger portion of shadow compared to layover. This would be the case if all objects in the scene were detached and the phenomena caused by them would not 
interfere with each other. But in the test scene the ground distance in range direction between the objects is often small. This results in many mixed pixels where shadow and layover are both present.

(Table 2)

Especially for building analysis from InSAR data, the influence of layover on the elevation measurement has to be considered (Bolter and Leberl, 2000). Due to the signal mixture, the elevation data tend to be too small at layover locations (neglecting the noise influence). Only $43 \%$ of the roof area is not interfered by layover or shadow.

In order to estimate the improvement by a second measurement, a SAR image with perpendicular illumination from west was simulated. In the second case, the sensing direction coincides with the maximum of the main road orientations. In approximately $45 \%$ of the area, an unreliable measurement in one view can be compensated by the data of the other view. However, still about a third of the area remains unreliable.

Such simulations can be carried out for different aspect directions in order to determine the best aspect or set of aspects maximising the surface visibility of a special object class. In the same way a variation of the viewing angle can be considered. For the test scene Karlsruhe, 648 simulations with 72 aspects and 9 viewing angles (steps in $5^{\circ}$ ) were carried out (Soergel et al., 2002b). By combining the four optimal SAR acquisitions approx. 85\% of the roof areas could be sensed at least by one view.

5.2 Detection of locations of strong backscatter 
For the detection of possible locations of double-bounce and total reflection phenomena a combined raster and vector ground truth is analysed. A detail of this hybrid ground truth is depicted in Fig. 8. In order to derive the vector data from the LIDAR DEM a building expectation area was determined from the building footprints. In this area planes were fitted to the elevation data (Stilla and Jurkiewicz, 1999).

(Figure 8)

Vertical planes oriented towards the sensor which are not occluded by shadow are candidates for double-bounce scattering (Meyer and Roy, 2000). Total reflection occurs if the normal vector of a plane points to the sensor. The detected candidates for corner structures shown in Fig. 9b agree well with superstructures on the rooftops in the aerial image (Fig. 9a) and bright stripes in the real SAR data (Fig. 9c). The shift of elevated objects towards the sensor is illustrated in the SAR simulation shown in Fig. 9d.

(Figure 9)

Particularly interesting is the rippled roof structure on the left, causing strong signal response. This example illustrates that strong scatterers cannot be detected from the building footprints alone. Hence, detailed building models should be incorporated for analysis of SAR data in urban areas.

\section{Conclusion and outlook}


It was shown that from InSAR data a segmentation of building structures is possible. The InSAR test dataset Frankfurt contained large and detached buildings with flat roofs. Extended building parts with different height could be distinguished by the segmentation approach. Due to the noisy nature of the InSAR elevation data, the achieved level of detail was not comparable to results from LIDAR. Furthermore, in dense build-up areas mutual interference of the signals from buildings may hinder building reconstruction. In spite of these limitations, InSAR can offer the opportunity to sense urban areas even in case of bad weather conditions, if LIDAR is not operable. A detection of areas with unreliable SAR data by incorporating apriori acquired elevation data is useful for analysing SAR images. 3D city models containing man-made objects in vector representation (CAD) which were combined with elevation data of natural objects (e.g. vegetation) in raster representation (DEM) were shown to be a wellsuited scene reference for this task.

The optimal viewing angle (off-nadir) depends on the specific scene section and can not be given in general. But, both the best aspect and viewing angle can be determined by simulations.

A combined analysis of SAR data from different aspects can enlarge the portion of reliable data. The evidence of the final results can be improved by mutual confirmation of segmentation results in each image. Techniques for the fusion of knowledge from the different InSAR surveys has to be further studied and is subject of future work.

\section{Acknowledgment}


We want to thank Dr. Ender (FGAN-FHR Research Institute for High Frequency Physics and Radar Techniques) for providing us the InSAR data (AER II).

\section{References}

Bolter, R., Leberl, F., 2000. Phenomenology-based and interferometry-guided building reconstruction from multiple SAR images. Proc. EUSAR, pp. 687-690.

Ender, J.H.G., 1998. Experimental results achieved with the airborne multi-channel SAR system AER-II. Proc. EUSAR, pp. 315-318.

Gamba, P., Houshmand, B., 2000. Digital surface models and building extraction: A comparison of IF-SAR and LIDAR data. IEEE Trans. on Geoscience and Remote Sensing 38(4), 1959-1968.

Hoepfner, K.B., 1999. Recovery of Building Structure from IFSAR-Derived Elevation Maps. Technical Report 99-16, Computer Science Department, University of Massachusetts, Amherst.

Kakumoto, S., Hatayama, M., Kameda, H., Taniguchi, T., 1997. Development of disaster management spatial information system. Proc. GIS'97 Conf., pp. 595-598.

Meier, E., Frei, U., Nuesch, D., 1993. Precise terrain corrected geocoded images. In: Schreier, G. (Ed.), SAR Geocoding: Data and Systems, Wichmann, Karlsruhe, pp. 173-185.

Meyer, R.H., Roy R.J., 2000. Algorithms for interpreting SAR imagery of complex building scenes. In: Zelnio, E. (Ed.), Algorithms for synthetic aperture radar imagery VII, SPIE Proc. Vol. 4053, pp. 642-651.

Schreier, G., 1993. Geometrical properties of SAR images. In: Schreier, G. (Ed.), SAR geocoding: data and systems, Wichmann, Karlsruhe, pp. 103-134.

Slatton, K.C., Crawford, M.M., Evans, B.L., 2000. Combining interferometric radar and laser altimeter data to improve estimates of topography. Proc. IGARSS (on CD ROM). 
Soergel, U., Thoennessen, U., Gross, H., Stilla, U., 2000. Segmentation of interferometric SAR data for building detection. International Archives of Photogrammetry and Remote Sensing, Vol. 33, part B1, pp. 328-335.

Soergel, U., Schulz, K., Thoennessen, U, Stilla, U., 2002a. Utilization of 2D and 3D information for SAR image analysis in dense urban areas. Proc. 4th European conference on synthetic aperture radar (EUSAR 2002), VDE, Berlin, pp. 429-434

Soergel, U., Thoennessen U., Stilla, U., 2002b. Utilization of LIDAR DEM for SAR Image Analysis in Dense Urban Areas. In: Kalliany, R., Leberl, F. (Eds.), Photogrammetric Computer Vision. International Archives of Photogrammetry and Remote Sensing, Vol. 34, Part 3B, pp. 255-258.

Stilla, U., Jurkiewicz, K., 1999. Reconstruction of building models from maps and laser altimeter data. In: Agouris, P., Stefanidis, A. (Eds.), Integrated spatial databases: Digital images and GIS, Springer, Berlin, pp. 34-46.

Takeuchi, S., Suga, Y., Yonezawa, C., Chen, C.H., 2000. Detection of urban disaster using InSAR - a case study for the 1999 great Taiwan earthquake. Proc. IGARSS (on CD ROM). 


\section{Figure captions}

Fig. 1. SAR image of test area Karlsruhe (City center, Castle and Campus).

Fig. 2. SAR system AER II, geometry of across-track interferometry, and signal processing.

Fig. 3. Projection of a building into a) slant image (IS) and b) ground image (IG).

Fig. 4. Processing chain for segmentation of buildings from InSAR data.

Fig. 5. a) oblique view of the scene, b) SAR image (ground range), c) vertical view, d) SAR image overlaid with the layer BUILDING of the vector map. Range direction from right to left.

Fig. 6. Geometric constraints for the acquisition of SAR images of buildings (see explanations in text).

Fig. 7. a) Laser elevation data, b) shadow simulation, c) layover simulation, d) SAR phenomena image with shadow and layover, e) simulation of SAR image, f) SAR image. Range direction from top to bottom.

Fig. 8. Subset of the combined raster and vector ground truth.

Fig. 9. a) aerial image, b) building footprints (grey) and locations of possible strong scattering (black), c) real SAR image, d) simulated SAR image with building footprints. 


\section{Table captions}

Table 1

Features of state-of-the-art LIDAR and InSAR systems

Table 2

Results of shadow/layover detection in per cent 


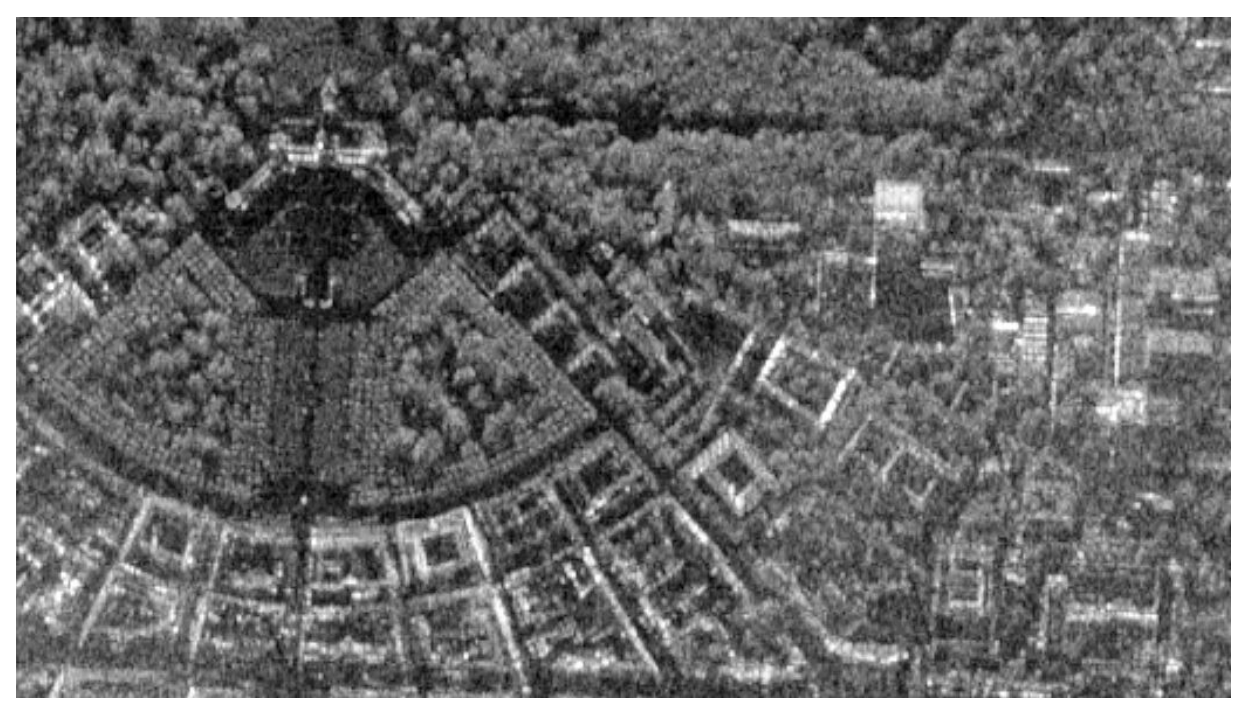

Fig. 1 

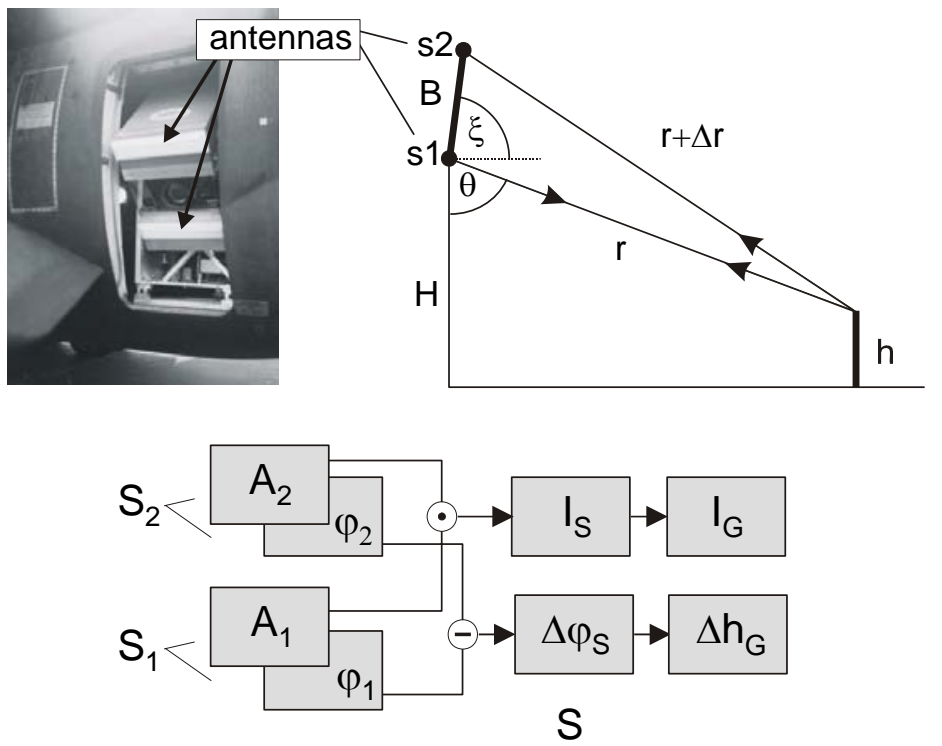

Fig. 2 

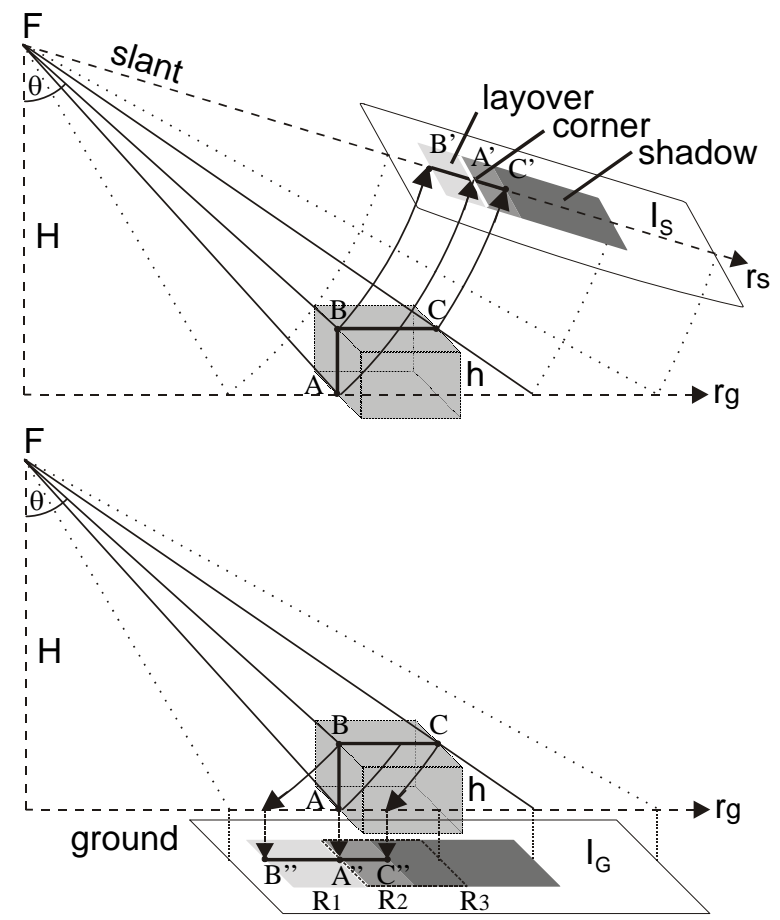

Fig. 3 


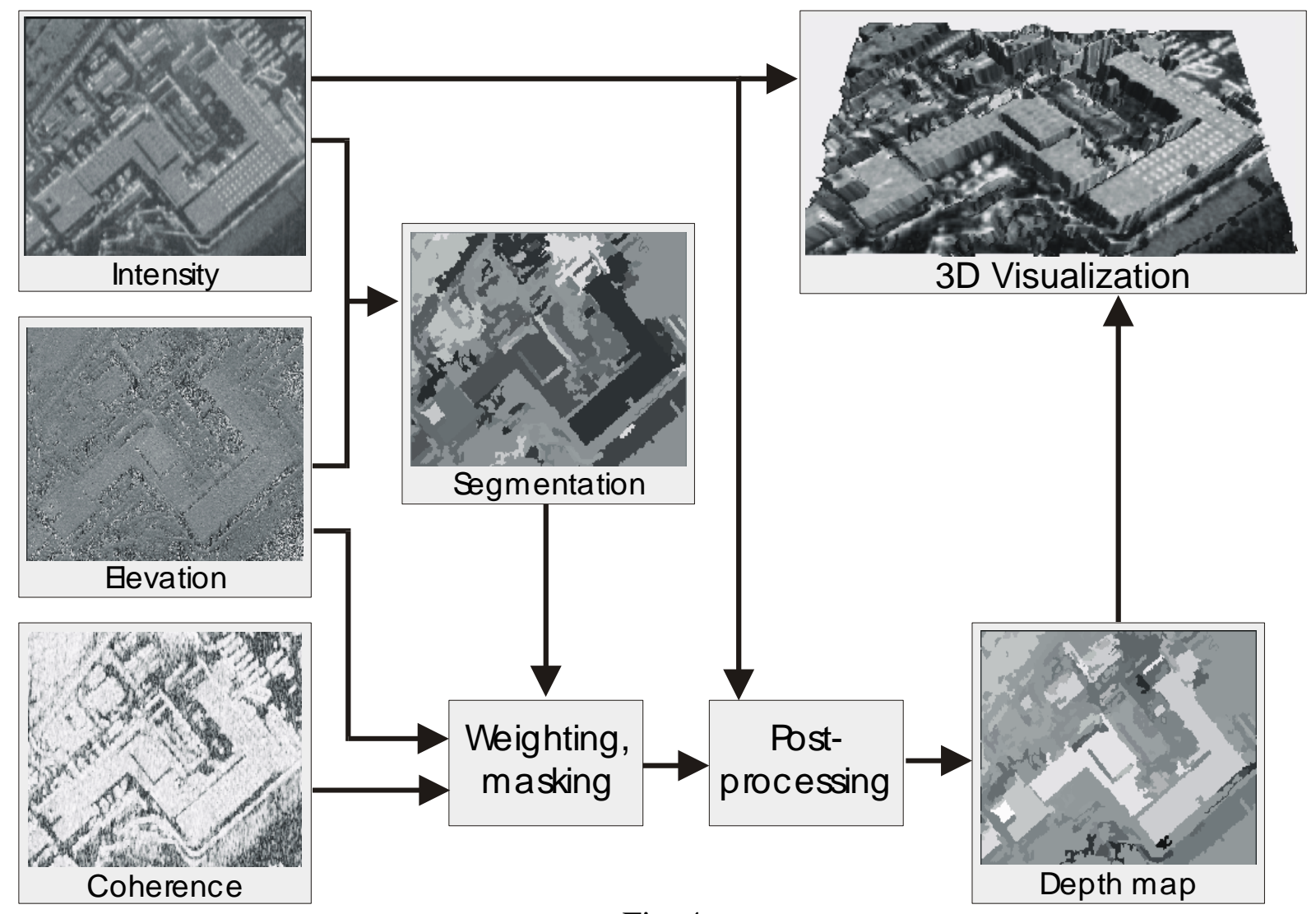

Fig. 4 
(a)

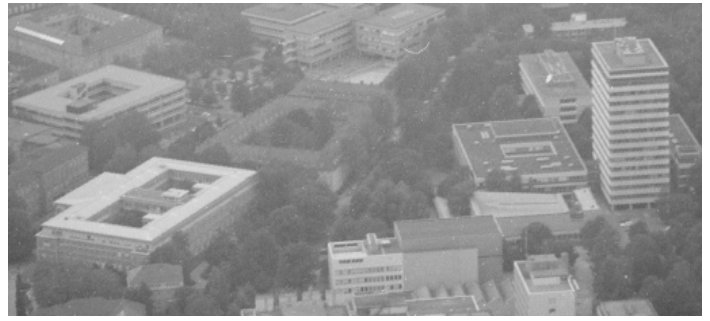

(b)
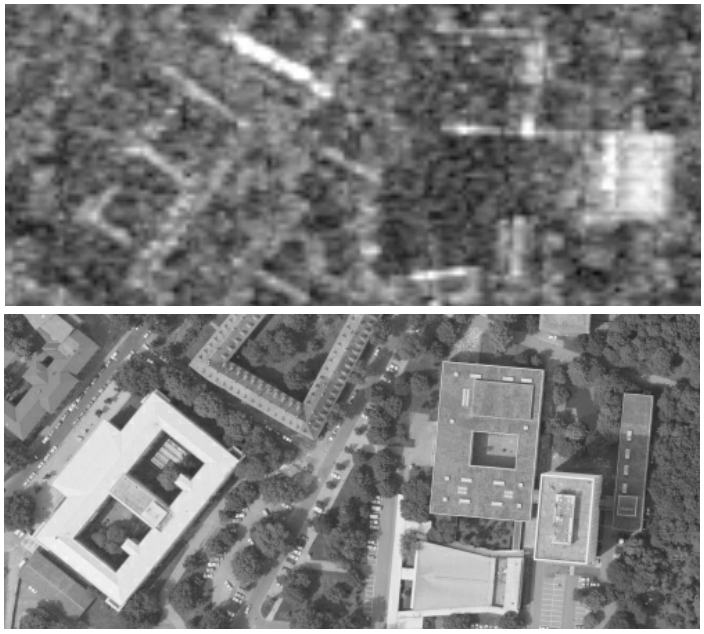

(c)

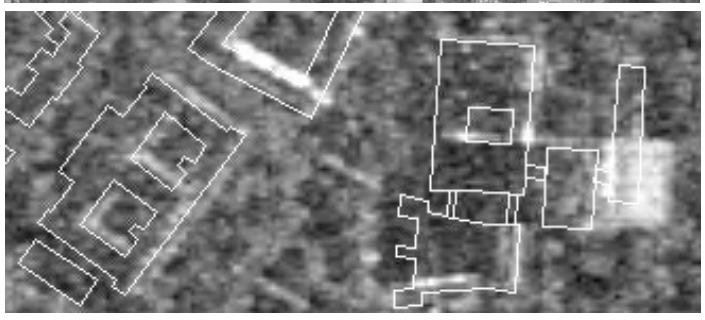

Fig. 5 


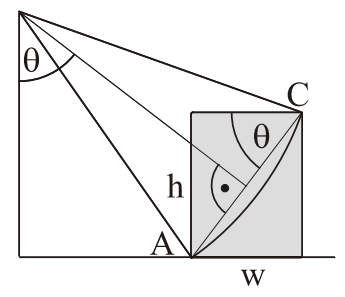

(a)

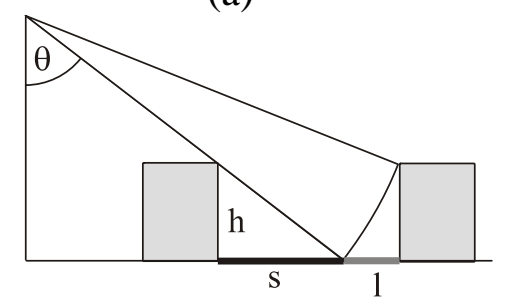

(c)

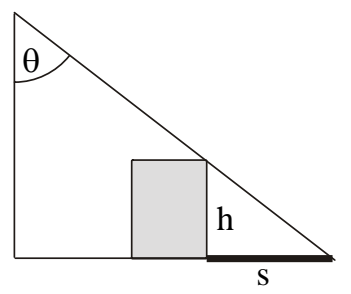

(b)

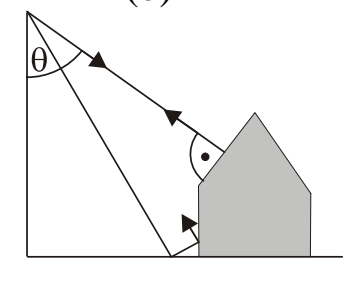

(d)

Fig. 6 


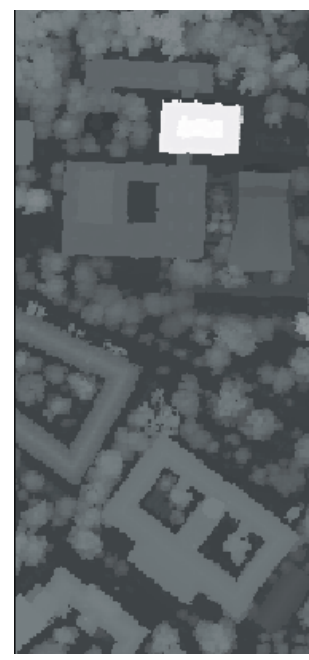

(a)

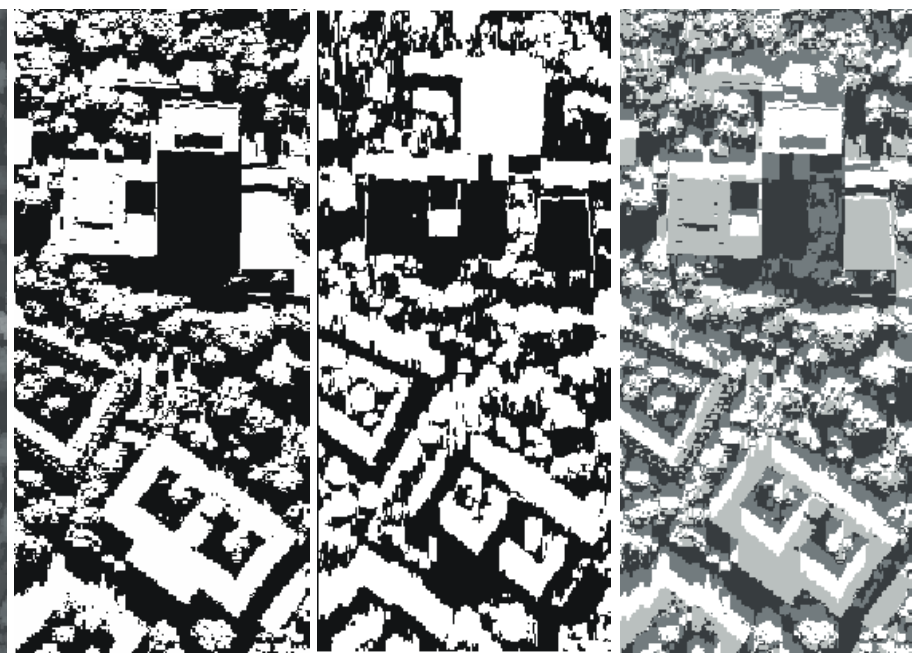

(c)

(b)

(d)

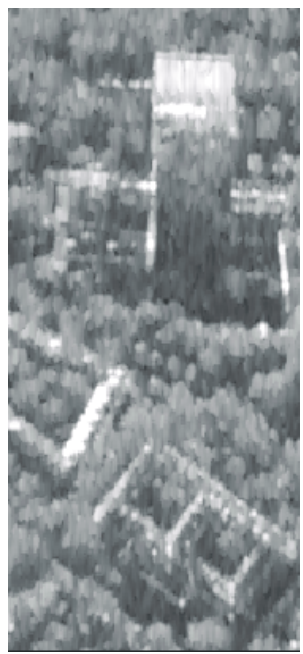

(e)

Fig. 7 


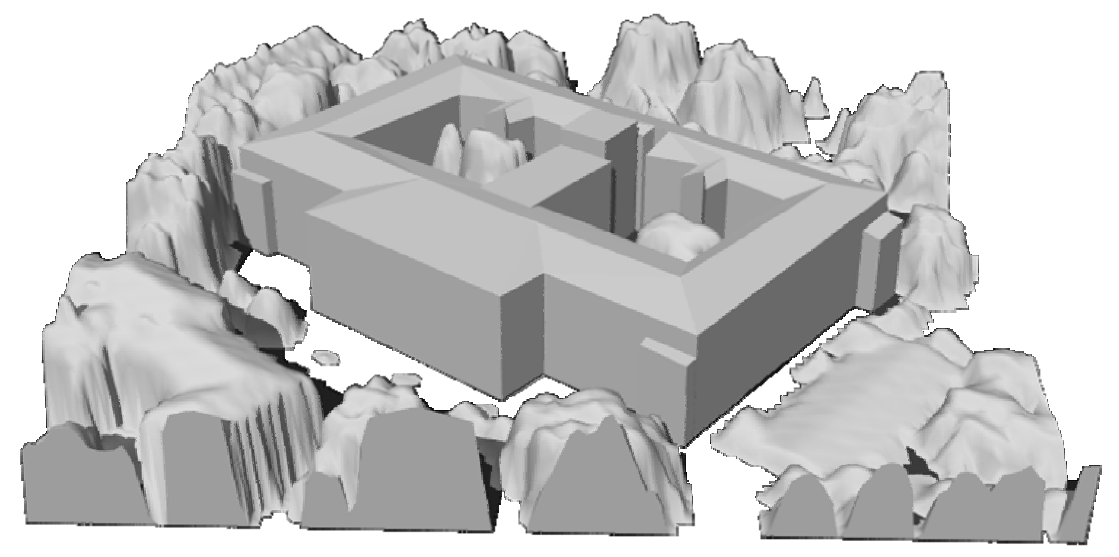

Fig. 8 

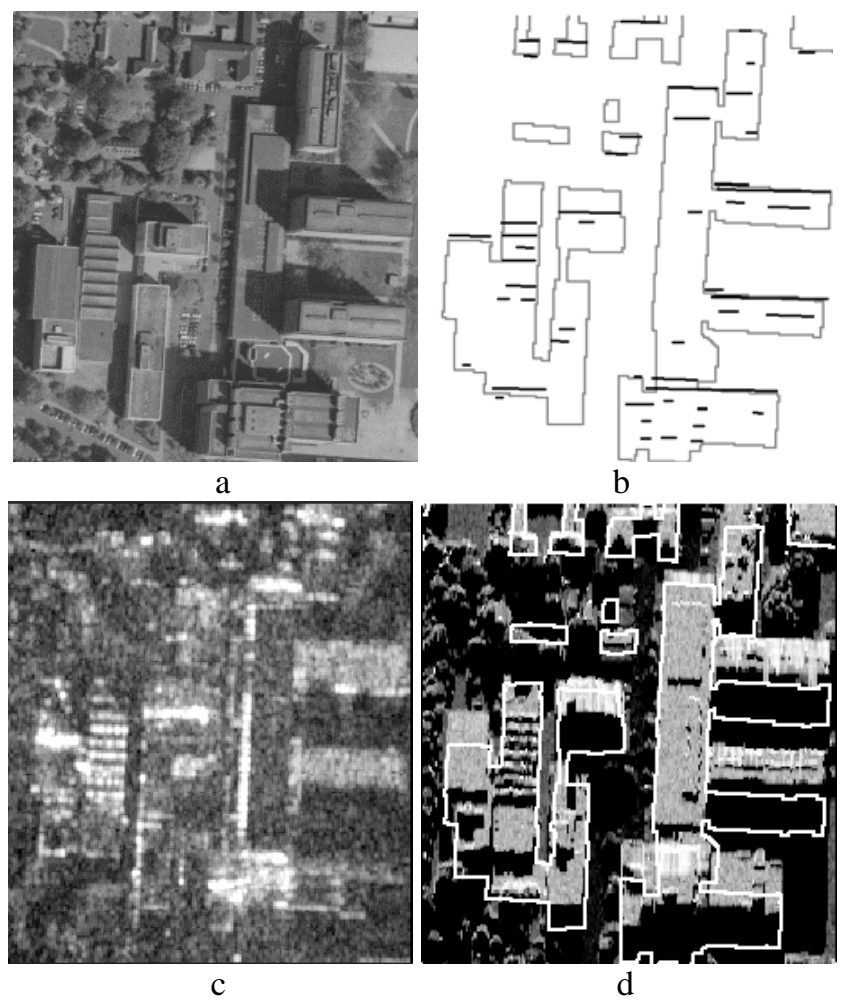

Fig. 9 


\begin{tabular}{|l|l|l|}
\hline & LIDAR & InSAR \\
\hline Signal & $\begin{array}{l}\text { reflected infrared } \\
\text { pulse }\end{array}$ & $\begin{array}{l}\text { two radar cross } \\
\text { sections }\end{array}$ \\
\hline Measurement & time-of-flight & phase difference \\
\hline Wavelength & $\mu \mathrm{m}$ & cm \\
\hline Illumination & nadir or side-looking & side-looking \\
\hline Range & $\begin{array}{l}\text { Attenuation in } \\
\text { atmosphere limits } \\
\text { range }\end{array}$ & $\begin{array}{l}\text { range and } \\
\text { weather } \\
\text { independent }\end{array}$ \\
\hline Elevation accuracy & dm & m \\
\hline Pixel resolution & dm-m & dm-m \\
\hline
\end{tabular}

Table 1 


\begin{tabular}{|l|c|c|c|}
\hline & $\begin{array}{c}\text { Complete } \\
\text { scene }\end{array}$ & Roads & $\begin{array}{c}\text { Building } \\
\text { roofs }\end{array}$ \\
\hline Shadow & 28 & 38.5 & 16 \\
\hline Layover & 25 & 18.5 & 33.5 \\
\hline Mixed & 19 & 23.5 & 7.5 \\
\hline Reliable & 28 & 19.5 & 43 \\
\hline
\end{tabular}

Table 2 